Original Research Paper

\title{
Development of Human-Computer Interactive Interface for Intelligent Automotive
}

\author{
Lyu Jianan', Ashardi Bin Abas ${ }^{2}$ \\ ${ }^{1}$ Huizhou University, China. \\ ${ }^{2}$ Universiti Pendidikan Sultan Idris, Malaysia.
}

Article History

Received:

01.10 .2020

Revised:

16.10.2020

Accepted:

16.11.2020

*Corresponding Author:

Ashardi Abas

Email:

ashardi@fskik.upsi.edu.my

This is an open access article, licensed under: $\mathrm{CC}-\mathrm{BY}-\mathrm{SA}$

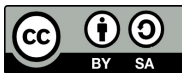

Abstract: The wide application of information technology and network technology in automobiles has made great changes in the Human-Computer Interaction. This paper studies the influence of Human-computer interaction modes on driving safety, comfort and efficiency based on physical interaction, touch screen control interaction, augmented reality, speech interaction and somatosensory interaction. The future Human-com-puter interaction modes such as multi-channel Human-computer interaction mode and Human-computer interaction mode based on biometrics and perception techno-logy are also discussed. At last, the method of automobile Humancomputer interaction design based on the existing technology is proposed, which has certain guiding significance for the current automobile Humancomputer interaction interface design.

Keywords: Automobile Interactive Interface, HUD Augmented Reality Interaction, Multi-Channel Interaction Mode. 


\section{Introduction}

With the development of vehicle network technology and information technology, the interaction interface of automobile becomes richer and more diversified. Automobile is not only a traditional means of transportation, but also a personal space for information acquisition, transmission, communication and entertainment [1]. At present, the information model has gradually developed from a single driving and condition information model to a complex information system including automobile information, inter-car information (car to car), information of car and other information carriers (car to $\mathrm{x})[2]$.

In such a complex information system, besides completing the main driving task (primary task) of controlling the car, maintaining the lane and monitoring the road condition, drivers also perform a large number of in-vehicle secondary tasks which have nothing to do with driving or are not directly related to driving. These secondary tasks will occupy the driver's visual, cognitive and action resources to some extent, and distract the driver's attention and produce a higher cognitive load [3].

Many studies have proved that secondary tasks represented by in-vehicle information interaction seriously affect driver's driving efficiency and traffic safety [4] [5]. The guidelines issued by the National Highway Transportation Commission in 2013 require that drivers should not spend more than 2 second on any operation [6].

Facing with the increasingly complex automobile interaction system, how to make users understand the hierarchical relationship of information clearly, how to get information nodes clearly, how to process information quickly and efficiently, how to improve operation efficiency, so as to perform operation accurately and efficiently, is the starting point of automobile Human-computer interaction design research [7].

\section{Current Situation of Automotive Human-Computer Interactive Interface Design}

\subsection{Physical Interaction Mode}

Traditional vehicle Human-computer interaction mainly uses knobs, buttons and other related equipments (such as brakes, etc) in the form of physical interaction. After more than 100 years of development, the physical form of Human-computer interaction has been very mature, and is considered to be the most reliable and efficient way of Human-computer interaction [8].

Most of the functions can be accessed at the touch of physical keys, such as switching airconditioning, which can be opened by pressing AC or heating keys. At the same time, physical buttons respond quickly and can be recognized and feedback instantly. At the same time, they can be adjusted quickly and accurately by rotating knobs, such as adjusting the volume of multimedia, and the temperature and air volume of air-conditioning accurately, etc.

With the introduction of intelligent traffic system (ITS) and intelligent control system (ICS), new functions are pouring into automobiles. Interaction mode based on physical buttons is facing great challenges. A typical case is the increasing number of physical operators such as keys and knobs, as shown Figure 1 (Buick Oncora).

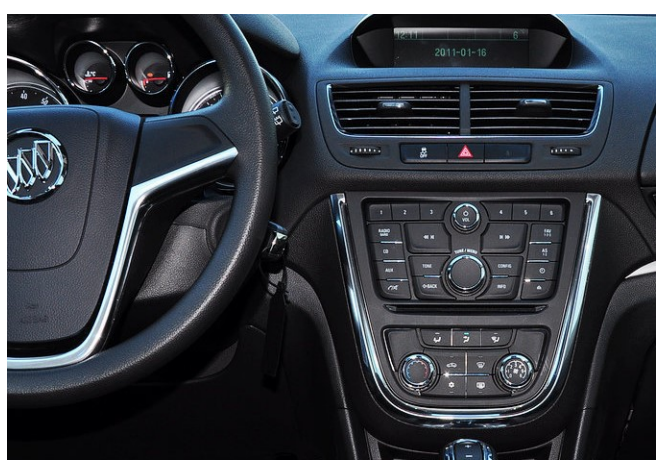

Figure 1. Buick Oncora

The increasing physical operators lead to difficulties in recognition, reduce cognitive and operational efficiency, and even affect driving safety. On the limited physical interaction interface, the increasing keys will affect the aesthetic perception of the central console on the one hand, on the other 
hand, it will reduce the volume of keys, thus affecting the operational feeling. In addition, physical keys will cause mechanical failure after long-term using.

\subsection{Touch Screen Control Interactive Mode}

Owing to the increasing new functions and the insurmountable shortcomings of physical operators, the interaction mode based on physical operators is facing enormous challenges, which makes touch control develop rapidly.

As early as 1989, Buick Riviera first used a monochrome touch screen to control radio and air conditioning functions on the screen, as shown in Figure 2 (Buick Riviera).

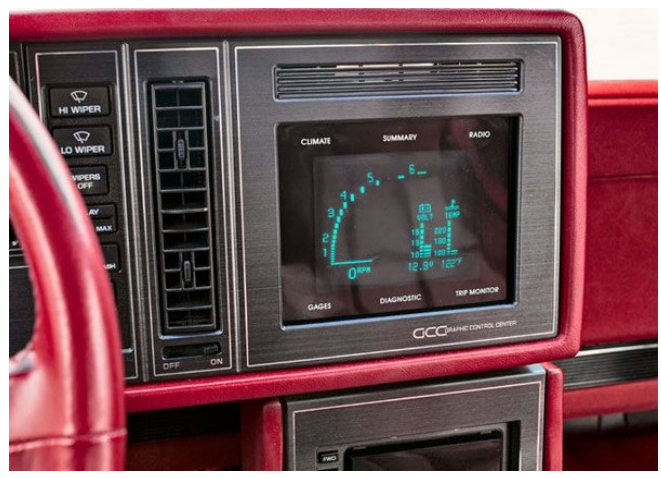

Figure 2. Buick Riviera

In 2013, the use of touch screens in Tesla's Model S reached its peak. The central control area of Model S was occupied by a big whole touch screen. The whole car had only a few necessary physical keys on the steering wheel and door panel, as shown in Figure 3 (Tesla model S).

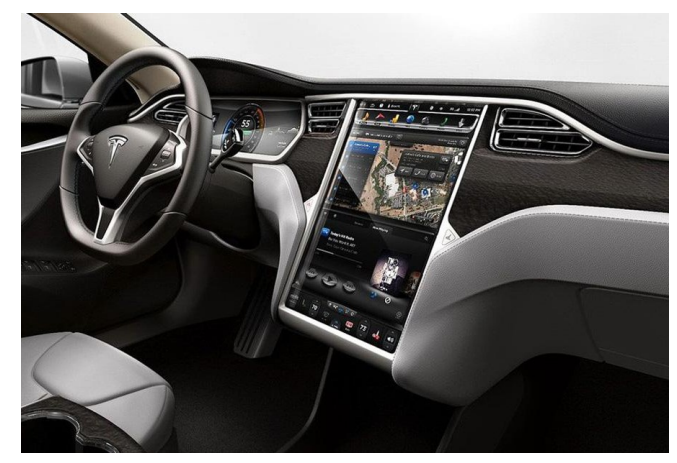

Figure 3. Tesla Model S

Touch screen not only makes car interior more concise, but also has a strong sense of technology compared with physical buttons. Touch screen has the following advantages:

(a) Multi-point touch (more than two points) can realize various operations such as enlargement and reduction.

(b) Without physical keys, software can also be used to implement various operations, such as zooming, moving, etc.

(c) The operation object displayed is identical with the input object and has direct sense operability.

(d) The integration of input and display can minimize the machine.

(e) There will be no gaps like switches and keys, preventing entering garbage, dust, water, etc. 
However, touch interaction has obvious limitations in the process of Human-computer interaction. Firstly, there are many layers of touch interaction, which lead to lower operation efficiency. For example, in order to adjust the temperature, we first need to turn on the air-conditioning interface, then select the cold and warm icon, and then adjust the temperature, each click to increase or decrease 0.5 degrees.

Secondly, touch operation cannot achieve rapid and precise adjustment. For example, it is difficult to accurately and efficiently adjust the temperature of air-conditioning and the volume of multimedia.

Thirdly, when the environmental factors such as temperature and humidity change, the performance of capacitive touch screen will be unstable and even drift. When the finger is wet (such as sweat, etc), or when the touch screen is foggy, it will lead to the failure of touch operation; moreover, some touch screens have no feedback during touch operation, which can easily lead to distraction. Drivers need to stare at the screen for touch operation. In other words, touch screen is not conducive to blind operation. To drivers who need to concentrate on driving will face enormous risk.

\subsection{Speech Interaction Mode}

Speech interaction refers to the direct use of speech as a sensor to directly control equipments without the need for physical controllers. The command of speech interaction does not have the hierarchical relationship required by touch control, so speech control can reduce the driver's operation steps by reducing the operation steps and improving work efficiency.

Speech and speech interaction can keep the driver's vision forward, and both hands do not need to leave the steering wheel, thus improving safety, as shown in Figure 4. Therefore, one of the best interfaces is the Speech-User Interface (SUI). It is well adapted to people with reduced mobility and to some emergency situations (hands-free and distant interaction) $[9,10,11]$.

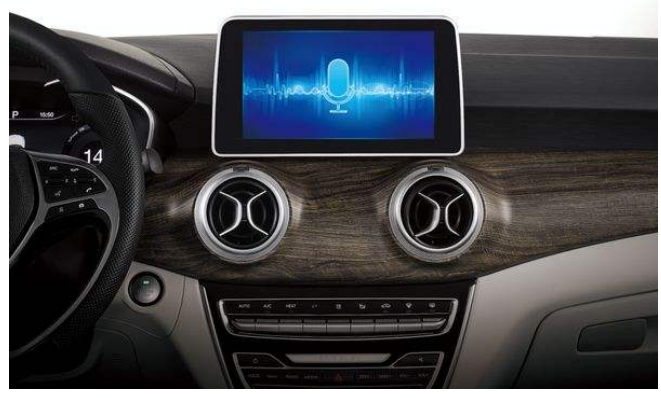

Figure 4. Speech interaction

At present, the speech recognition accuracy is as high as 95\% [12], but in practice, speech recognition is affected by many factors, which reduces its accuracy.

- Firstly, it is difficult to understand grammar and semantics in the process of speech input, especially in the case of polysemy.

- Secondly, in the process of speech recognition, dialect has a great impact on the recognition efficiency.

- Thirdly, in speech input, the context will cause different understanding of letters and words, thus bringing differences to the recognition. In addition, the accuracy of speech recognition is also affected by tone, environmental noise and interference. At this stage, the first problem to be solved in speech interaction is to improve speech recognition technology and semantic recognition technology.

\subsection{Somatosensory Interaction Technology}

Somatosensory interaction technology is an intelligent technology that can directly use limb movements to interact with digital devices and environment without any control equipment, and complete various instructions according to human actions, and manipulate them at will.

In the process of sensory interaction, users can naturally make corresponding actions according to the situation and needs, and control the device without thinking too much about the details of the operation. In other words, natural sensory interaction reduces the complexity of manipulation, and 
enables users to focus more on the semantics expressed by actions and the content of interaction. Gesture recognition function in BMW iDrive system (intelligent driving control system), which detects gesture movements through 3D sensors, controls infotainment functions in an intuitive and convenient way, as shown Figure 5.

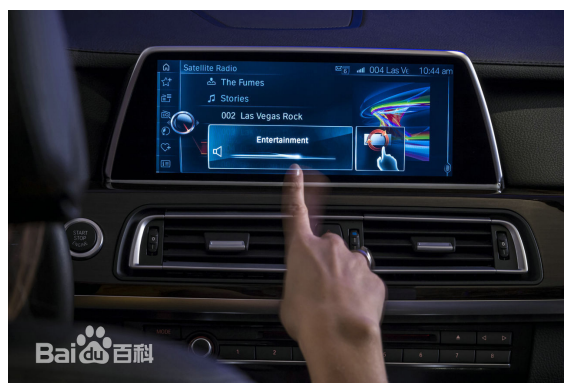

Figure 5. Gesture interaction

The essence of somatosensory interaction technology is to use hand as the carrier of gesture recognition, and control the light switch, infotainment programs and temperature, etc. in the car through different gestures.

However, this mode of operation requires the driver's hand to leave the steering wheel to perform different gestures, leading to the driver cannot concentrate on the driving behaviour itself, increasing the interference of non-driving factors, thus increasing the driving insecurity.

\subsection{HUD Visual Augmented Reality Technology}

In the field of automotive Human-computer interaction, head-up display (HUD) and augmented reality (AR) are combined to overlay the virtual information and real world information on the screen and interact on this basis, as shown Figure 6.

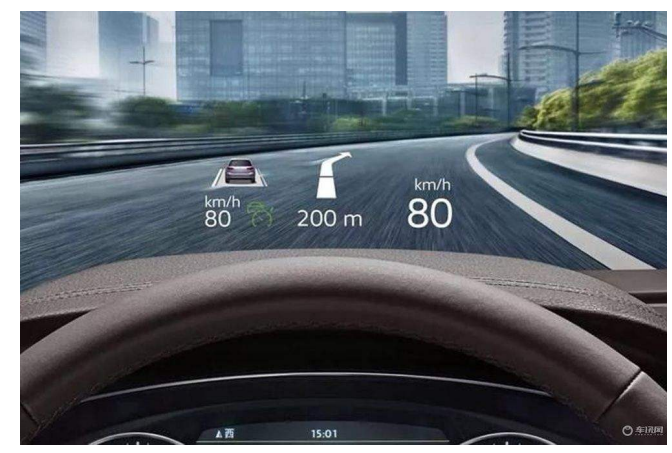

Figure 6. HUD AR Visual Studio

Head-up display (HUD) projects navigation information, speed and safety warning information to the windshield, and generates virtual graphics to overlap with the real driving environment. HUD augmented reality technology allows drivers not to look down at the display information on the instrument, but to maintain a head-up posture, reduce the frequency of looking down at the instrument, avoid attention interruption and loss of mastery of situation awareness.

In addition, the head-up display system will display warning information from various driving assistance systems, such as lane departure warning, pedestrian avoidance warning from night vision system with pedestrian recognition function, etc. HUD visual augmented reality technology plays an important role in vehicle safety. 


\section{Development Trend of Human-Computer Interaction}

\subsection{Interaction Model Based on Biometrics and Perception Technology}

The safety is a major demand for automobile drivers for a long time and in the future. The development of biometrics and perception technology enhances the intelligent perception ability of automobiles, and provides a guarantee for further improving driving safety.

On the basis of personal identification and combined with physiological and psychological perception technology, intelligent vehicle can realize real-time monitoring of people's physiological and psychological state. After the recognition system is identified as fatigue driving, the information is sent to the automobile control system by wireless (Wi-Fi). After that, the automobile intelligent control system alarms and wakes up the driver through speech, and at the same time, controls the car to turn on the danger warning lights and alarm the surrounding vehicles, as shown in Figure 7.

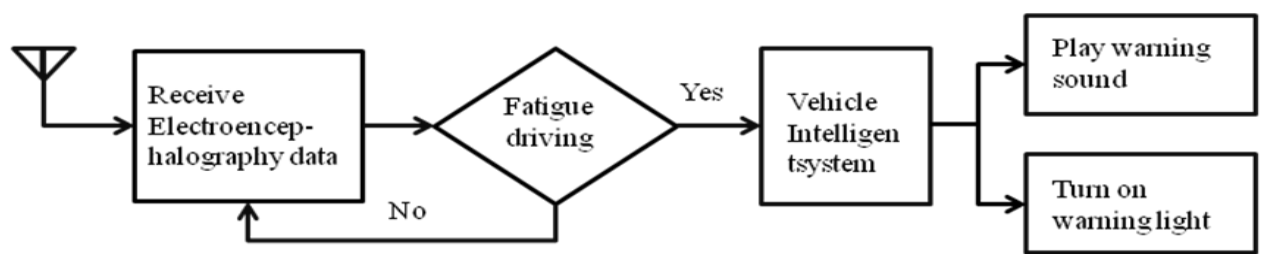

Figure 7. Automatic Alarm Schematic Diagram

In this way, it can not only protect the driver's safety to the greatest extent, but also prompt other vehicles to avoid the car. Human-computer interaction technology based on biometrics and perception technology will bring important guarantee to driving safety. This Human-computer interaction mode will be a very important design and research topic.

\subsection{Multichannel Human-Computer Interaction Mode}

The main forms of traditional Human-computer interface for automobiles are display screen, physical keys, steering wheel and other related equipments (such as hand brake, brake and throttle, etc.). In recent years, with the development of speech recognition technology, sensing techno-logy, machine sensory enhancement technology and emotional computing techno-logy, it provides technical conditions for the realization of multi-channel human-computer interaction. Future forms of interaction will no longer rely too much on visual information, but will emphasize the combination of multi-sensory channels [13].

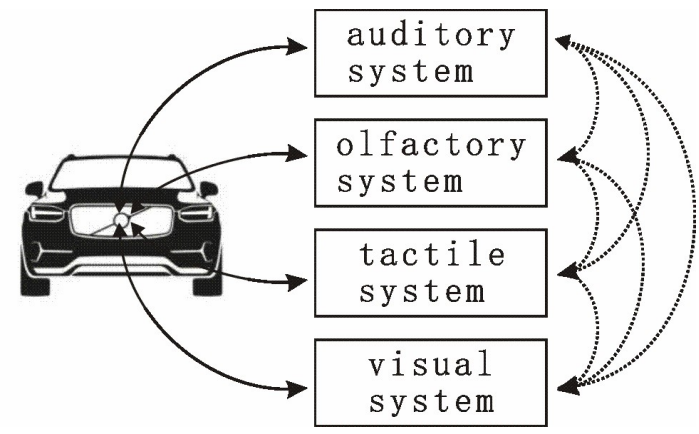

Figure 8. Multi-Channel Interaction Mode

Multi-channel Human-computer interaction is an important feature of the user experience of intelligent vehicles, which will give users a new experience in safe driving, work efficiency and sensory experience, as shown in Figure 8.

At present, the accuracy of speech recognition has reached $95 \%$. With the further maturity of speech interaction technology, the accuracy rate reaches $99 \%$. Speech technology will play an 
important role in reducing drivers' distraction, improving interaction efficiency and driving safety. Speech interaction will become an important interactive mode of Human-computer interaction in intelligent vehicles. Gesture interaction scheme has good interesting and attractive in automobile environment [14].

The combination of gesture interaction and other interaction methods will solve the problems of system recognition requirements and driving safety in the process of gesture recognition. The interaction of olfactory channel is the most possible way to achieve new interaction in the future, which will provide drivers with new sensory experience.

Multi-channel combination is the key to the realization of multi-channel Human-computer interaction mode. The core advantage of multi-channel Human-computer interaction mode is to transcend different single perception channel and achieve integration with other different channels.

\section{Suggestions on Design of Interactive Interface for Intelligent Vehicles}

Intelligent touch screen makes interior look concise, and makes interior get strong sense of technology and fashion. The use of intelligent touch screen in automobile has become a trend. As analyzed in the previous 2.2, touch interaction in automotives has some drawbacks that are difficult to overcome at present.

Therefore, full touch screen is not the best design of vehicle interaction at present [15]. Some automobile manufacturers began to return to physical control mode gradually for some function control. Speech control realizes natural communication in a complete sense [16], and is considered as the safest way of Human-computer interaction.

Speech technology plays an important role in reducing driver distraction, improving interaction efficiency and driving safety. Because speech recognition is influenced by many factors, such as dialect and environmental noise, further breakthroughs in speech recognition and semantic recognition are needed to achieve a complete Human-computer dialogue-recognition accuracy of $99 \%$.

In order to adapt to the development trend of touch screen and overcome the problems of physical interaction, touch interaction and speech interaction, the design of automobile Human-computer interaction should adopt multi-channel hybrid interaction mode, and assign different functions to different interaction modes, so as to give full play to the respective advantages of touch control, speech control and physical control.

The Human-computer interaction design of automobiles should follow the following principles:

(a) Adjust the conditioner's wind speed, temperature and multimedia volume through the physical controller, without needing to control by repeatedly clicking the screen or long pressing the screen.

It does not need to be adjusted by clicking on the screen many times or pressing the screen for a long time.

Setting the shortcut keys of the best multimedia volume and the most suitable airconditioning temperature and air volume so as to be accessed by one click to achieve accurate and efficient operation.

(b) If touch-screen control is necessary, it should follow a flat layout principle to minimize the layers, one-click access to direct operation without complex menu pages.

(c) Speech control can shield some error recognition results and reduce unreasonable control caused by error recognition. At the same time, the speech control system needs to set oneclick to start and close to prevent the audio signal input of non-user instructions.

(d) Using augmented reality technology, made the important driving information such as navigation project to the front windshield to reduce the driver's line-of-sight deviation and improve driving safety.

\section{Conclusion}

Automotive Human-computer interaction interface is an important platform for automobile and driver. Friendly Human-computer interaction interface is an important guarantee for safe, comfortable and efficient Human-computer interaction between automobile and driver.

Therefore, automobile Human-computer interaction design has always been a very important research topic. With the rapid development of information technology and network technology, more 
intelligent Human-computer interaction technologies, interactive equipments and interactive interfaces will provide more secure, efficient and comfortable driving experience.

This paper discusses the design and research of automobile Human-computer interaction interface from three aspects: the current situation of automobile Human-computer interaction technologies, the developing direction of automobile Human-computer interaction interface and the design of interaction mode, and describes the current situation of the development of academia and industry in this field.

At present, the applications of many new Human-computer interaction technologies in the automobile field are still in the experimental stage, and there are many technical bottlenecks. It is reasonable to believe that the future Human-computer interaction interface will bring people a better life and driving experience.

\section{References}

[1] L. Weijie, "Research on interactive design of automotive digital instrument panel," Design, pp. 138-140, November 2018

[2] A. Schmidt, W. Spiessl, and D. Kern, "Driving Automotive User Interface Research [J]," Journal of IEEE Pervasive Computing, no. 1-3, pp. 85-88, 2010.

[3] J. S. Wang, R. Knipling and M.J. Goodman, "The Role of Driver Inattention in Crashes: New Statistics from the 1995 Crashworthiness Data System," Proceeding of the 40th Annual Conference of the Association for the Advancement of Automotive Medicine, Vancouver, Canada: [s.n.], pp. 377-392, 1996

[4] D. L. Strayer and F.A. Drews "Profiles in Driver Distraction: Effects of Cell Phone Conversations on Younger and Older Drivers," Journal of Human Factors, vol. 46, no. 4, pp. 640-649, 2004.

[5] S.J. Kass, K. S. Cole, and C. J. Stanny, "Effects of Distraction and Experience on Situation Awareness and Simulated Driving," Journal of Transportation Research Part F: Traffic Psychology and Behavior, vol.10, no. 4, pp. 321-329, 2007.

[6] Anonymity, "The Next Battlefield for Automobile Manufacturers: "flatting" of Central Control Console [J]," Journal of Agricultural equipment and vehicle engineering, vol. 53, no. 1, pp. 6, 2015.

[7] P. W. Sun, J. M. Yang, and Sun Yuan-bo, "Research on hierarchical design of automobile human-computer interface," Journal of Mechanical design, vol. 36, no. 2, pp. 121-125, 2019.

[8] T. Hao, Z. Jianghong, and W. Wei, "Research on human-computer interaction interface design for automobiles," Journal of automotive engineering, vol. 2, no. 5, pp. 315-321, 2012.

[9] L. Haihua, "Research on Key Technologies of Intelligent Human-Computer Interaction Assistant Based On Voice Control," Computer Products and Circulation, pp. 103-105, December 2017.

[10] S. Bouakaz, M. Vacher, M-E. Bobillier-Chaumon, F. Aman, S. Bekkadja, F.O. Portet, E. Guillou, S. Rossato, E. Desser'ee, P. Traineau, J-P. Vimon, and T. Chevalier, "CIRDO: Smart companion for helping elderly to live at home for longer," Innovation and Research in Biomedical Engineering, vol. 35, no. 2, pp. 101-108, February 2019. doi: http://dx.doi.org/10.1016/ j.irbm. [Accessed: February 1, 2019].

[11] M. Vacher, S. Caffiau, F. O. Portet, B. Meillon, C. Roux, E. Elias, B. Lecouteux, and P. Chahuara. "Evaluation of a context-aware voice Interface for Ambient Assisted Living: qualitative user study vs quantitative system evaluation," ACM Trans. Access. Comput. 7, 2, Article, vol. 5, no. 36, May 2015.

[12] H. Menglong, L. Yunhong, M. Liping, Z. Xiaoni, and W. Gangyi, "Design of intelligent home voice control system based on ARM," Journal of Computer knowledge and technology, vol. 14, no. 34, pp. 178-180, 2008.

[13] M. Chai, S. W. Li, and W. C. Sun, "Drowsiness Monitoring Based on Steering Wheel Status [J]," Journal of Transportation Research Part D: Transport and Environment, 2018.

[14] A. S. M. Mahfujur Rahman, J. Saboune, and A. E. Saddik, "motion-path based in car gesture control of the multimedia devices," Symposium of the first ACM international symposium on Design and analysis of intelligent vehicular networks and applications, Miami, Florida, USA. November 2011 [Online]. doi: 10.1145/2069000.2069013. [Accessed: June 2019]. 
Lyu Jianan, Ashardi Bin Abas.

Development of Human-Computer Interactive Interface for Intelligent Automotive.

International Journal of Artificial Intelligence, vol. 7, no. 2, pp. 13-21, December 2020. DOI: 10.36079/lamintang.ijai-0702.134

[15] P. Yuyuan and L. Jialiang, "Research on interactive design of vehicle-mounted infotainment system based on Internet of vehicles," Electronic World, vol. 20, pp. 136-137, 2018.

[16] C. Yue and S. Qijun, "Development trend of intelligent voice technology and application of telecom operators," Posts and telecommunications design technology, pp. 6-11, December 2016. 